\title{
A Combination of Exercise and Therapy with Cabergoline Attenuate Disturbances of Pituitary-Gonadal Hormones in Hyperprolactinemic Male Patients
}

\author{
Mohammad Fayiz AbuMoh'd', Samir Qasim', Nesreen Bataineh², Loay Salman³ \\ Affiliations: 'Yarmouk University, Faculty of Physical Education, Department of Sports/Movement Sciences, Irbid, \\ Jordan, ${ }^{2}$ Yarmouk University, Faculty of Medicine, Department of Basic Medical Sciences, Irbid, Jordan, ${ }^{3} J o r d a n$ \\ University of Science and Technology, Faculty of Medicine, Department of Basic Medical Sciences, Irbid, Jordan
}

Correspondence: M. F. AbuMoh'd, Yarmouk University, Faculty of Physical Education, Department of Sports/Movement Sciences, University St., Irbid, Jordan. E-mail: famohammad@yu.edu.jo

\begin{abstract}
This study aimed to investigate whether cabergoline therapy alone for six months or in combination with a light exercise programme for an additional three months can attenuate hyperprolactinemia in 13 male patients (range: 22 to 45 yrs.) through measuring pituitary-gonadal hormones including prolactin, follicle-stimulating hormone, luteinizing hormone, and total testosterone. The exercise programme consisted of walking, brisk walking, jogging, and running for three months, during which the intensity and duration of exercise were gradually increased. All the patients performed the exercise programme at $6.30 \mathrm{AM}$ to exclude the effects of the circadian rhythm. The exercise programme was performed at an intensity below 160 beats per min to ensure filling of the heart with blood during ventricular diastole. Blood samples were collected from each patient on three occasions: before treatment, at the end of cabergoline therapy, and after the combination of light exercise and cabergoline. At the end of cabergoline therapy, descriptive data revealed that serum prolactin levels were decreased while the other hormones were increased but without returning to the normal range, except for one patient. However, at the end of the combination procedure, most hormones, namely prolactin and testosterone, were returned to the normal range in most patients. In conclusion, light exercise combined with cabergoline therapy for additional 3 months after 6 months of cabergoline therapy alone returned hormones in most patients to normal range probably due to improved mood and decreased hostility as a result of tuberoinfundibular pathway activity.
\end{abstract}

KEY WORDS hypogonadism, hypoglycaemia, tuberoinfundibular pathway, acetylcholine, galactorrhoea

@MJSSMontenegro

EXERCISE, CABERGOLINE, AND HYPERPROLACTINEMIA

http://mjssm.me/?sekcija $=$ article\&artid $=201$

\section{Introduction}

Hyperprolactinemia is defined as the presence of abnormally high levels of serum prolactin (PRL) levels of above $20 \mathrm{ng} / \mathrm{ml}$ in men and above $25 \mathrm{ng} / \mathrm{ml}$ in women (Majumdar \& Mangal, 2013). It affects the pituitary-gonadal axis function (Kulshreshtha et al., 2017; Toulis et al., 2018) through impaired binding of gonadotropins to the testes, which causes hypogonadism (Laufer, Margalioth, Livshin, Ben-David, \& Schenker, 1981). Hypogonadism may decrease spermatogenesis (Panidis, Rousso, Skiadopoulos, Panidou, \& Mamopoulos, 1997), resulting in reduced libido, impotence (EL-Beheiry, Souka, EL-Kamshoushi, Hussein, \& EL-Sabah, 1988; Sperling \& Bhatt, 2016), and anorgasmia.

Hyperprolactinemia is mainly caused by pituitary adenomas (i.e., prolactinomas) (Toulis et al., 2018). However, it can also be triggered by other etiopathological cases including liver disease (Laufer et al., 1981), thyroid disorders (Tirgar-Tabari, Sharbatdaran, Manafi-Afkham, \& Montazeri, 2016; Sharma, Dutta, \& Sharma, 2017), psychopharmacological drugs (Alpak et al., 2014; Kilic, Ozturk, Deveci, \& Nrpinar, 2018; Palubska et al., 2017;

Received: January 202020 | Accepted after revision: March 152020 | First published online: Septembar 012020

(c) 2020 by the author(s). License MSA, Podgorica, Montenegro. This article is an open access article distributed under the terms and conditions of the Creative Commons Attribution (CC BY).

Conflict of interest: None declared. 
Raveendranthan et al., 2018; Yancar-Demir \& Sayin, 2014), and antidepressants (Kilic et al., 2018). In addition, hyperprolactinemia is related to a high-protein diet and hypoglycaemia (Palubska et al., 2017). Furthermore, stressful situations may also induce hyperprolactinemia (EL-Beheiry et al., 1988; Palubska et al., 2017).

Hyperprolactinemia can be asymptomatic (Alpak et al., 2014; Majumdar \& Mangal, 2013) or be associated with clinical manifestations such as oligomenorrhea, amenorrhea, galactorrhoea (Dogan et al., 2016; Sperling \& Bhatt, 2016), erectile dysfunction, gynecomastia (Alpak et al., 2014; Raveendranthan et al., 2018; Yancar-Demir \& Sayin, 2014), oligospermia, azoospermia (Laufer et al., 1981), and infertility (Alpak et al., 2014; Tirgar-Tabari et al., 2016). Hence, there is an urgent need to treat hyperprolactinemia. Hyperprolactinemia can be inhibited by somatostatin, acetylcholine, norepinephrine (Majumdar \& Mangal, 2013), gamma-aminobutyric acid (GABA) (Majumdar \& Mangal, 2013; Nishimura et al., 1999), and dopamine (Gulleroglu et al., 2012; Khare et al., 2017; Nishimura et al., 1999; Yancar-Demir \& Sayin, 2014). Significantly, the blockage of the tuberoinfundibular has been shown to cause hyperprolactinemia, since the activity of this pathway transmits dopamine from the arcuate nucleus to the pituitary gland, through which it inhibits the release of prolactin (Inci Kenar \& Sozeri Varma, 2014). Cabergoline (CAB), a dopamine receptor agonist, can suppress PRL secretion (Dogan et al., 2016; Gulleroglu et al., 2012; Inci Kenar \& Sozeri Varma, 2014; Khare et al., 2017; Raveendranthan et al., 2018; Sperling \& Bhatt, 2016) and reduce adenoma size in the patients with prolactinomas (Dogan et al., 2016; Khare et al., 2017). CAB is associated with side effects, however, such as nausea, dizziness, nasal stuffiness, vomiting, headache (Majumdar \& Mangal, 2013), blurred vision, and male sexual dysfunction (Buckworth, Dishman, O'Connor, \& Tomporowski, 2013). Moreover, a recent research study reported that it might be unsafe, especially in cardiac valves, if the ingested dose is more than $3 \mathrm{mg}$ a week (Khare et al., 2017). Dogan et al. (2016) demonstrated an increased risk of cardiovascular diseases (CVD) in patients with prolactinoma who suffered from hyperprolactinemia during therapy with a dopamine agonist.

Previous research reported a positive effect of dopamine receptor agonists on the normalization of PRL levels but not follicle-stimulating hormone (FSH), luteinizing hormone (LH), and total testosterone (TT) (Ishikawa, Kaneko, Ohashi, Nakagawa, \& Hata, 1993). In addition, the treatment process with CAB for prolactinoma lasts for a relatively long duration (Ghadirian, Shirani, Ghazi-Mirsaeed, Mohebi, \& Alimohamadi, 2018). Indeed, Sperling and Bhatt (2016) have shown no effects of 10 months of CAB therapy $(0.25 \mathrm{mg}$ twice per week) on PRL normalization. For this reason, alternative treatment methods could be examined, specifically those considering that the exercise intervention could attenuate depressant and hostility (Oldham et al., 2009) and suppress mood swings, which are all associated with elevated PRL levels. Moreover, since there is a link between stress and hyperprolactinemia, exercise may play a crucial role in stress reduction through exercise-induced dopamine release.

To the best of our knowledge, no study has been conducted to evaluate the pituitary-gonadal hormones by a combination of light exercise and $\mathrm{CAB}$ therapy in hyperprolactinaemic male patients. Therefore, this study aimed to investigate whether $\mathrm{CAB}$ therapy alone for six months or combined with light exercise for three months attenuate hyperprolactinemia in male patients by measuring pituitary-gonadal hormones, including PRL, FSH, LH, and TT. We hypothesized that CAB would decrease PRL levels, but a combination of CAB and light exercise would have a significant effect, probably due to activated blood circulation, thereby decreasing the elevated PRL concentration.

\section{Methods}

Participants

Thirteen male patients $(\mathrm{n}=13)$ with hyperprolactinemia within the age range of 22 to 45 years (mean \pm SD: $34.46 \pm 7.22$ years) who visited the endocrinology outpatient clinics between February 2015 and October 2017 were evaluated endocrinologically. Five patients were married, and one of them was affected by macroprolactinoma. Patients were treated with different doses of CAB depending on their PRL levels. Thyroid function and 25-OH-Vitamin D were normal in all patients. The characteristics of patients ( age, BMI, CAB dose, and duration of disease) are shown in Table 1.

\section{Procedure}

One day prior to the beginning of the study procedure, blood samples $(8 \mathrm{ml})$ were collected from the median vein at 8:30 AM after overnight fasting (approximately 10 hours) to determine the baseline measurements (Table 2). Six months after CAB therapy alone, blood samples were collected following the same procedure during the baseline measurement. Additionally, in agreement with the physicians, CAB dose was roughly reduced to half after completion of the six months, based on each patient's dose. After that, all patients were involved in a light exercise programme for another three months. Each participant voluntarily provided written informed consent before participation in the present study. This study was approved in advance by the local scientific research committee (SS101-2019).

\section{Exercise}

The exercise programme consisted of walking, brisk walking, jogging, and running for three months, during which the intensity and duration of exercise were gradually increased (Table 3). The exercise was performed by all the patients at 6.30 AM to exclude the effects of their circadian rhythm and to ensure sufficient excre- 


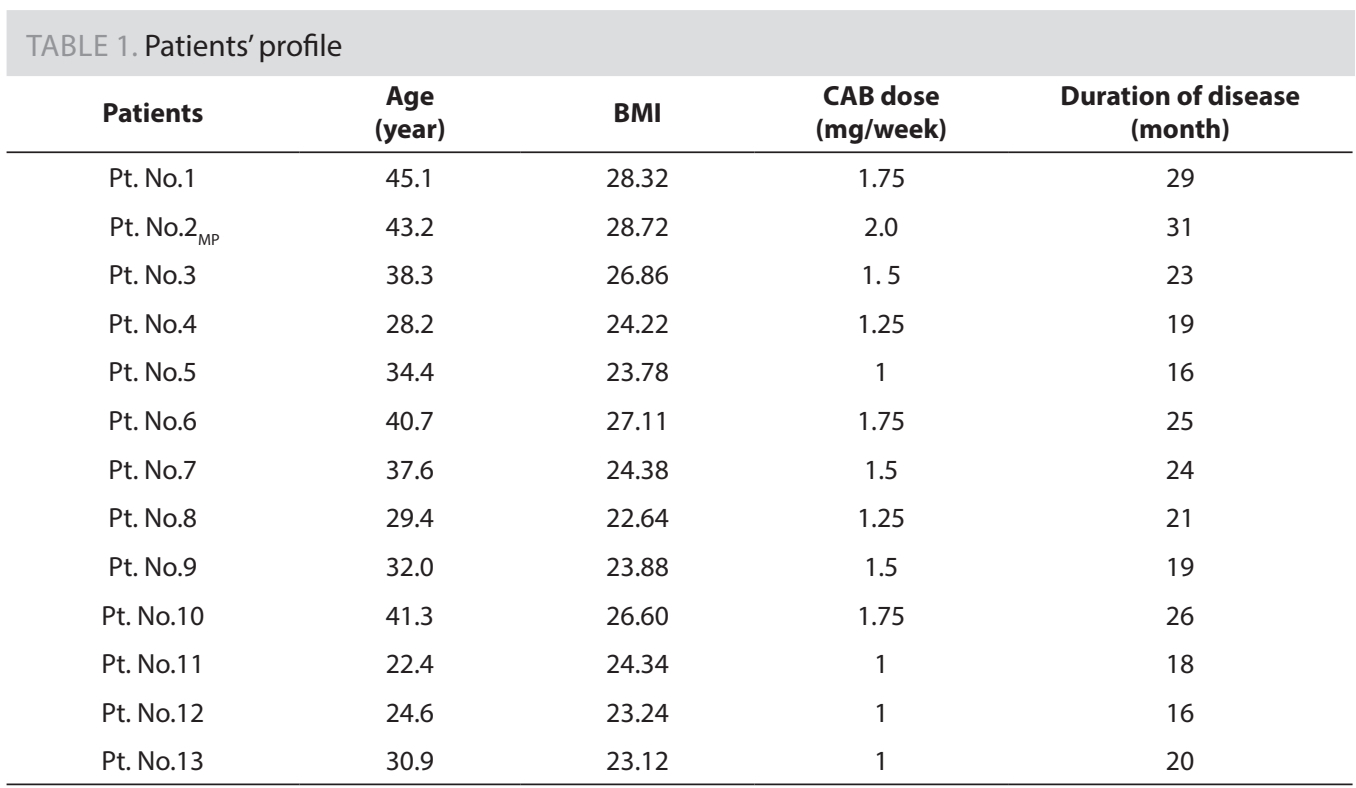

Note. Pt. No. - Patient number; BMI - Body mass index; CAB - Cabergoline; MP - affected by macroprolactinoma.

tion of cortisol and thyroid hormones, which are responsible for the regulation of the body's metabolic ratio. The exercise was performed at an intensity below 160 beats per min to ensure the filling of the heart with blood during ventricular diastole. Heart rate (HR) was monitored by a pulse monitor (Samsung, Gear Sport). During these three months, the patients were asked to engage in either a bicycle ride or amusement swimming every Friday. Each patient ate an apple half an hour before the start of each exercise session for energy supply. At the end of the exercise programme, blood samples were collected and analysed for the hormonal responses. The exercise programme was designed during piloting with a well-trained athlete who experienced hyperprolactinemia for 24 months. During his disease, he was never engaged in any type of exercise due to general weakness and lack of energy.

After completing all tests, according to the social validation concept, the first author conducted focus group interviews with the patients. Social validation is designed to ensure that the programme considers views from the patients perspective (Barker et al., 2013) and to evaluate the satisfaction with a programme. During this stage, we mainly focused on the participants' satisfaction of the programme as it has been previously suggested (Page \& Thelwell, 2013).

TABLE 2. Baseline measurements of the patient's pituitary-gonadal hormones

\begin{tabular}{ccccc} 
Patients & $\begin{array}{c}\text { PRL (ng/ml) } \\
\text { (NR: } \mathbf{4 . 0 - 1 5 . 2 0 )}\end{array}$ & $\begin{array}{c}\text { FSH (mlU/ml) } \\
\text { (NR: } \mathbf{1 . 5}-\mathbf{1 2 . 4 0})\end{array}$ & $\begin{array}{c}\text { LH (mlU/ml) } \\
\text { (NR: 1.7 - 8.6) }\end{array}$ & $\begin{array}{c}\text { TT (ng/dl) } \\
\text { (NR: 249 - 836) }\end{array}$ \\
\hline Pt. No.1 & 563 & 0.59 & 0.15 & 25.9 \\
Pt. No.2 & 835 & 0.18 & 0.1 & 18.7 \\
Pt. No.3 & 370 & 1.01 & 0.33 & 38 \\
Pt. No.4 & 222 & 0.35 & 0.19 & 112 \\
Pt. No.5 & 85 & $2.6^{*}$ & $2.1^{*}$ & 202 \\
Pt. No.6 & 484 & 0.36 & 0.82 & 27.5 \\
Pt. No.7 & 300 & 1.15 & 0.49 & 44 \\
Pt. No.8 & 267 & 1.22 & 0.88 & 96 \\
Pt. No.9 & 282 & 1.37 & 0.45 & 67 \\
Pt. No.10 & 445 & 0.95 & 0.28 & 22.2 \\
Pt. No.11 & 58 & $2.2^{*}$ & $2.6^{*}$ & $262^{*}$ \\
Pt. No.12 & 188 & $1.8^{*}$ & 1.3 & 166 \\
Pt. No.13 & 176 & 0.61 & 1.61 & 144 \\
\hline
\end{tabular}

Note. Pt. No. - Patient number; PRL - Prolactin; FSH - Follicle-stimulating hormone; LH - Luteinizing hormone; TT - Total testosterone; * - Hormone value within normal range; NR - normal range.

All quantitative data were presented descriptively due to the wide range of the participants' characteristics, such as CAB dosage and PRL levels. Therefore, following the previous research (Barker et al., 2013; Buckworth et al., 2013), we only presented absolute changes in PRL, FSH, LH, and TT. The qualitative data, however, was reported verbatim as told by patients. 
Prolactin, follicle-stimulating hormone, luteinizing hormone, and total testosterone were stored in a plain tube, centrifuged at $4500 \mathrm{RPM} / 10 \mathrm{~min}$ and quantitated by radioimmunoassay (Cobas E411; Roche, Germany).

\begin{tabular}{|c|c|c|c|c|}
\hline \multirow[t]{2}{*}{ Month 1} & Week 1 & Week 2 & Week 3 & Week 4 \\
\hline & 8 min walking & $\begin{array}{l}8 \text { min walking } \\
2 \text { min brisk walking }\end{array}$ & $\begin{array}{l}3 \text { min walking } \\
3 \text { min brisk walking }\end{array}$ & $\begin{array}{l}10 \text { min walking } \\
4 \text { min brisk walking }\end{array}$ \\
\hline \multirow[t]{2}{*}{ Month 2} & Week 1 & Week 2 & Week 3 & Week 4 \\
\hline & $\begin{array}{l}10 \text { min walking } \\
5 \text { min brisk walking } \\
1 \text { min jogging }\end{array}$ & $\begin{array}{l}11 \text { min walking } \\
6 \text { min brisk walking } \\
1 \text { min jogging }\end{array}$ & $\begin{array}{l}12 \text { min walking } \\
8 \text { min brisk walking } \\
1 \text { min jogging }\end{array}$ & $\begin{array}{l}12 \text { min walking } \\
8 \text { min brisk walking } \\
2 \text { min jogging }\end{array}$ \\
\hline \multirow[t]{2}{*}{ Month 3} & Week 1 & Week 2 & Week 3 & Week 4 \\
\hline & $\begin{array}{l}10 \text { min walking } \\
10 \text { min brisk walking } \\
4 \text { min jogging }\end{array}$ & $\begin{array}{l}10 \text { min walking } \\
15 \text { min brisk walking } \\
1 \text { min running }\end{array}$ & $\begin{array}{l}10 \text { min walking } \\
16 \text { min brisk walking } \\
2 \text { min running }\end{array}$ & $\begin{array}{l}14 \text { min walking } \\
6 \text { min brisk walking } \\
4 \text { min jogging } \\
6 \text { min running }\end{array}$ \\
\hline
\end{tabular}

\section{Results}

Table 4 illustrates the results of pituitary-gonadal hormones responses to CAB therapy alone for six months, followed by three months of light exercise combined with CAB therapy.

\section{CAB therapy}

Data revealed that serum PRL levels were decreased while the other hormones were increased but without returning to normal range, except one patient No. 11 who had his hormones back to normal range compared to the other 12 patients. Of relevance, patient No. 5 had FSH, LH, and TT back to the normal range but without any decreasing in the PRL level. FSH and LH levels were decreased to the normal range in patients No. 12 and 13. The FSH level was in the boarder lower limit in Patient No. 9.

\section{Exercise and $C A B$}

Data revealed that most patients returned their hormones, especially PRL and TT to normal range. However, Patients No. 1, 2, 6, and 10 failed to lower their hormones to the normal range. Significantly, Patient No. 4 had normal TT and LH levels but did not experience a decrease in PRL and an increase in FSH levels. Although PRL levels were abnormally high in Patients No. 3, 4, and 7, these levels are still considered to be less than 20 $\mathrm{ng} / \mathrm{ml}$; therefore, they were not near the point of hyperprolactinemia.

TABLE 4. Pituitary-gonadal hormones after 6 months of treatment with cabergoline therapy alone followed by 3 months of light exercise combined with cabergoline therapy

\begin{tabular}{|c|c|c|c|c|c|c|c|c|}
\hline \multirow{2}{*}{ Patients } & \multicolumn{4}{|c|}{ After therapy with CAB alone } & \multicolumn{4}{|c|}{ After a combination of light exercise and CAB therapy } \\
\hline & PRL & FSH & LH & TT & PRL & FSH & LH & TT \\
\hline Pt. No.1 & 175 & 0.9 & 0.39 & 67.4 & 49 & 1.17 & 0.5 & 157 \\
\hline Pt. No.2 & 282 & 0.66 & 0.25 & 49.7 & 67 & 0.98 & 0.49 & 134 \\
\hline Pt. No.3 & 61 & 1.35 & 0.69 & 97 & 19.2\# & 1.69 & $1.90^{*}$ & 230 \\
\hline Pt. No.4 & 57 & $1.17^{*}$ & 0.53 & 169 & $17.4 \#$ & 1.62 & $1.9^{*}$ & $281^{*}$ \\
\hline Pt. No.5 & 24.7 & $3.6^{*}$ & $2.4^{*}$ & $325^{*}$ & $8.8^{*}$ & $5.1^{*}$ & $3.8^{*}$ & $430^{*}$ \\
\hline Pt. No.6 & 133 & 0.51 & 1.1 & 74 & 33.6 & 1.29 & $2.77^{*}$ & 186 \\
\hline Pt. No.7 & 85 & 1.35 & 1.4 & 114 & 15.2\# & $2.08^{*}$ & $3.1^{*}$ & $294^{*}$ \\
\hline Pt. No.8 & 53 & 1.42 & 0.98 & 154 & $12.5^{*}$ & $2.25^{*}$ & $2.0^{*}$ & $331^{*}$ \\
\hline Pt. No.9 & 41 & $1.7^{*}$ & 1.02 & 133 & $14.8^{*}$ & $2.15^{*}$ & $1.9^{*}$ & $292^{*}$ \\
\hline Pt. No.10 & 96 & 1.29 & 0.55 & 69 & 27.1 & $1.87^{*}$ & 1.49 & 195 \\
\hline Pt. No.11 & $13.3^{*}$ & $2.5^{*}$ & $2.8^{*}$ & $319^{*}$ & $5.4^{*}$ & $5.01^{*}$ & $3.4^{*}$ & $482^{*}$ \\
\hline Pt. No.12 & 28.7 & $2.3^{*}$ & $1.9^{*}$ & 222 & $10.2^{*}$ & $3.1^{*}$ & $3.4^{*}$ & $400^{*}$ \\
\hline Pt. No.13 & 38.1 & $1.27^{*}$ & $2.09^{*}$ & 189 & $13.4^{*}$ & $2.03^{*}$ & $2.9^{*}$ & $373^{*}$ \\
\hline
\end{tabular}

Note. Pt. No. - Patient number; PRL - Prolactin; FSH - Follicle-stimulating hormone; LH - Luteinizing hormone; TT - Total testosterone; CAB - Cabergoline; * Hormone value within normal range; \# - refers to serum PRL levels which are less than $20 \mathrm{ng} / \mathrm{ml}$.

\section{Discussion}

This study investigated whether CAB therapy alone for six months or in combination with light exercise for an additional three months can attenuate hyperprolactinemia in male patients through measuring pituitary-gonadal hormones. We hypothesized that CAB would decrease PRL levels, but a combination of CAB and light 
exercise would have a significant effect, probably due to activated blood circulation thereby decreasing the elevated PRL concentration.

\section{CAB therapy}

This study revealed that the therapy with $\mathrm{CAB}$ alone alleviated the increased PRL levels and elevated the decreased FSH, LH, and TT in most of the patients. These results could be explained by the role of CAB in the activation of the tuberoinfundibular pathway, which regulates PRL secretion (Inci Kenar \& Sezeri Varma, 2014; Yancar-Demir \& Sayin, 2014) through dopamine binding to D2 receptors on lactotrophic cells (Alpak et al., 2014). The blockage of inhibitory neurons in the tuberoinfundibular pathway induces increased pituitary PRL secretion (Chapurin, Wang, Steinberg, \& Jang, 2016).

In addition, $\mathrm{CAB}$ therapy returned hormones to normal ranges in only one patient. This result might be attributed to insufficient treatment duration based on the baseline values, especially PRL and TT. Sperling and Bhatt (2016) have shown that serum PRL levels decreased from $974 \mathrm{ng} / \mathrm{ml}$ to $568 \mathrm{ng} / \mathrm{ml}$ after CAB therapy ( $0.25 \mathrm{mg}$ twice per week) in patients affected by pituitary macroadenoma $(3.2 \mathrm{~cm}$ measure in adenoma size). The authors found that PRL at 10 months follow-up was decreased to $290 \mathrm{ng} / \mathrm{ml}$, and thus they demonstrated the difficulty of attenuation in elevated serum PRL levels. Recently, Ghadirian et al. (2018) reported that $1 \mathrm{mg}$ of CAB per week returned serum PRL levels from $358 \mathrm{ng} / \mathrm{ml}$ to $21 \mathrm{ng} / \mathrm{ml}$ after one year with CAB therapy in patients with macroprolactinoma, associated with intertumoral haemorrhage in a follow-up visit. In addition, Patient No. 11 showed enhanced levels of all hormones that were returned to normal ranges. These results could be explained by the long duration of treatment based on the baseline values of his hormones, which were the lowest values among patients before starting the CAB therapy.

\section{Exercise and $C A B$}

Data revealed that most patients, except No. 4, exhibited normalized hormonal levels, which might be attributed to the effect of light exercise in mood enhancement. Hyperprolactinemia is affected by mood. In addition to this, exercise could lessen the aggressive feelings that result from hyperprolactinemia, keeping the patient away from daily stress. In this regard, El-Beheiry et al. (1988) reported that $12 \%$ of patients with hyperprolactinemia were predisposed to stressful situations prior to their diagnosis with hyperprolactinemia. In the present study, five patients reported that they had had stressful lives, especially when the diagnosis of hyperprolactinemia was idiopathic. After beginning regular exercise, however, the patients felt happier with less stress. For instance, Patient 6 said: "I feel happy and strong again". Patient 10 added: "My life and my sexual life became much better".

Exercise has a positive biological effect on the pituitary-adrenocortical axis (Oldham, Zimmerman, \& Hotfield, 2009); therefore, it can act as an inhibitor on disturbances that occur in the pituitary-gonadal axis. Another explanation by which exercise enhanced the mood of patients with hyperprolactinemia is that exercise induces excretion of $\beta$-endorphin, which is responsible for pain relief (Grossman \& Sutton, 1985); thus, it improves headache associated with hyperprolactinemia. It has been documented that brain $\beta$-endorphin, which is defined as a suppressor of central fatigue, attenuates psychological stress (Yamashita \& Yamamoto, 2014) and enhances social life. Furthermore, it has been shown that neurotransmitter dopamine is secreted at high levels during exercise (Abbiss \& Laursen, 2005); which may improve muscle activation and enhance mood. While dopamine increases during physical activity, it may elicit motivation and increase arousal (Barker, Mellaliey, McCarthy, Jones, \& Moran, 2013). For these reasons, we postulated that exercise might act as a "psychological booster" in hyperprolactinaemic patients.

It has been thought that very low-intensity exercise (e.g., jogging and brisk walking) could regulate the release of gonadotropin-releasing hormone $(\mathrm{GnRH})$ secreted from the arcuate nucleus (Bailey, Davis, \& Ahlborn, 1993; Abbiss \& Laursen, 2005). This hormone is suppressed in patients with hyperprolactinemia, resulting in decreased FSH and LH (Majundar \& Mangal, 2013; Sperling \& Bhatt, 2016). Unfortunately, no research has been conducted into the effect of exercise on the attenuation of hyperprolactinemia. Therefore, further research is needed.

Significantly, Patient No. 2 has been affected by macroprolactinoma. This case could be too complicated for returning his hormone values to normal range. Pathologically, a tumour may compress the optic chiasm, which causes blurred vision (Dogan et al., 2016; Toulis et al., 2018). Moreover, a tumour causes inhibition of tuberoinfundibular dopamine pathway (Chapurin et al., 2016), resulting in increased pituitary PRL release. The other patients whose hormonal values did not return to their normal range after exercise might need a longer duration of the exercises based on the higher hormonal values they had. While PRL potentiates the LH to ensure spermatogenesis in testes by direct action on the Leydig cells (Laufer et al., 1981; Nishimura et al., 1999), PRL also inhibits the action of LH while present in abnormally high levels in the blood, resulting in a reduction of testosterone production (Nishimura et al., 1999) and positive feedback to the hypothalamus, which induced the suppression of GnRH release.

Finally, it could be crucial that patients believe that they can overcome this challenge due to exercise, for example, Patient 8 said: "I think that such exercises should be introduced as a regular treatment. Physicians must use them to treat people with hyperprolactinemia [...].I am saying this from my experience. It really works". 
It is worth noting that those patients were overweight according to their BMI, which may indicate a link between high serum PRL levels and overweight or obesity. More research is needed regarding this. A limitation of the present study was that sperm count, sperm concentration, and sperm motility, as well as magnetic imaging resonance for the pituitary gland, were not measured.

\section{Conclusion}

In conclusion, light exercise combined with $\mathrm{CAB}$ therapy had a positive effect on the treatment of 13 male patients affected by hyperprolactinemia. This shows that light exercise combined with CAB therapy for additional three months after six months of CAB therapy alone returned prolactin, follicle-stimulating hormone, luteinizing hormone, and total testosterone in most patients to normal range probably due to improved mood and decreased hostility as a result of tuberoinfundibular pathway activity. We suggest introducing the light exercise programme to patients with hyperprolactinemia to enhance the other treatments efficacy further.

\section{Highlights}

- Light exercise, combined with CAB therapy, attenuates the high levels of serum PRL and increases the low levels of serum FSH, LH, and TT in male patients with hyperprolactinemia.

- Light exercise enhances mood and diminishes aggressive feelings in male patients with hyperprolactinemia.

- Light exercise combined with CAB therapy return PRL, FSH, LH, and TT to normal range in some patients with hyperprolactinemia.

\section{Acknowledgements}

The authors thank the physicians for their secret data about patients, MedLabs Consultancy Group for blood analysis, and the patients for their participation and effort throughout the study.

\section{References}

Abbiss, C. R., \& Laursen, P. B. (2005). Models to explain fatigue during prolonged endurance cycling. Sports Medicine, 35(10), 865-898. doi: 10.2165/00007256-200535100-00004

Alpak, G., Unal, A., Bulbul, F., Akosy, I., Demir, B., \& Svas, H. A. (2014). Hyperprolactinemia due to paliperdone palmitate and treatment with Aripiprazole. Bulletin of Clinical Psychopharmacology, 24(3), 253-256. doi: 10.5455/bcp.20131213042842

Bailey, S. P., Davis, J. M., \& Ahlborn, E. N. (1993). Neuroendocrine and substrate responses to altered brain 5-HT activity during prolonged exercise to fatigue. Journal of Applied Physiology, 74(6), 3006-3012. doi: 10.1152/jappl.1993.74.6.3006

Barker, J. B., Mellaliey, S. D., McCarthy, P. J., Jones, M. V., \& Moran, A. (2013). A review of single-case research in sport psychology 1997-2012: Research trends and future directions. Journal of Applied Sport Psychology, 25(1), 4-32. doi: 10.1080/10413200.2012.709579

Buckworth, J., Dishman, R. K., O'Connor, P. J., \& Tomporowski, P. D. (2013). Exercise psychology. 2nd edition. Champaign, IL: Human Kinetics.

Chapurin, N., Wang, C., Steinberg, D. M., \& Jang, D. W. (2016). Hyperprolactinemia secondary to allergic fungal sinusitis compressing the pituitary gland. Case Reports in Otolaryngology, 2016, 1-3. doi: $10.1155 / 2016 / 7260707$

Dogan, A. B., Arduc, A., Tuna, M. M., Nasiroglu, I. N., Isik, S., Berker, D., \& Guler, S. (2016). Evaluation of atherosclerosis after cessation of cabergoline therapy in patients with prolactinoma. Anatolian Journal of Cardiology, 16, 440-447. doi: 10.5152/AnatolJCardiol.2015.6416

EL-Beheiry, A., Souka, A., EL-Kamshoushi, A., Hussein, S., \& EL-Sabah, K. (1988). Hyperprolactinemia and impotence. Archives of Andrology, 21, 211-214. doi: 10.3109/01485018808986743

Ghadirian, H., Shirani, M., Ghazi-Mirsaeed, S., Mohebi, S., \& Alimohamadi, M. (2018). Pituitary apoplexy during treatment of prolactinoma with cabergoline. Asian Journal Neurosurgery, 13(1), 93-95. doi: 10.4103/1793-5482.181130

Grossman, A., \& Sutton, J. R. (1985). Endorphins: What are they? How are they measured? What is their role in exercise? Medicine \& Science in Sports \& Exercise, 17, 74-81.

Gulleroglu, K., Olgac, A., Bayrakci, U., Erdogan, O., Kinik, S. T., \& Baskin, E. (2012). Hyperprolactinemia as a rare cause of hypertension in chronic renal failure. Renal Failure, 34(6), 792-794. doi: $10.3109 / 0886022 X .2012 .672313$

Inci Kenar, A. N., \& Sozeri Varma, G. (2014). Hyperprolactinemia with Aripiprazole: a case report and review of the literature. Bulletin of Clinical Psychopharmacology, 24(3), 257-260. doi: 10.5455/bcp.20131021025639

Ishikawa, H., Kaneko, S., Ohashi, M., Nakagawa, K., \& Hata, M. (1993). Retrograde ejaculation accompanying hyperprolactinemia. Archives of Andrology, 30, 153-155. doi: 10.3109/01485019308987749

Khare, S., Lila, A. R., Patil, R., Phadke, M., Kerkar, P., Bandgar, T., \& Shah, N. S. (2017). Long-term cardiac (valvulopathy) safety of cebergoline in prolactinoma. Indian Journal of Endocrinology and Metabolism, 21(1), 154-159. doi: 10.4103/2230-8210.196010

Kilic, A., Ozturk, A., Deveci, E., \& Nrpinar, I. (2018). Development of hyperprolactinemia induced by the addition of Bupropion to Venlafaxine XR treatment. Bezmialem Science, 6, 150-152. doi: 10.14235/ 
bs.2018.1119

Kulshreshtha, B., Pahuja, I., Kothari, D., Sharma, N., Gupta, S., \& Mittal, A. (2017). Menstrual cycle abnormalities in patients with prolactinoma and drug-induced hyperprolactinemia. Indian Journal of Endocrinology and Metabolism, 21(4), 545-550. doi: 10.4103/ijem.IJEM_515_16

Laufer, N., Margalioth, E. J, Livshin, J., Ben-David, M., \& Schenker, J. G. (1981). Effect of bromocriptine treatment on male infertility associated with hyperprolactinemia. Archives of Andrology, 6, 343-346. doi: $10.3109 / 01485018108987547$

Majumdar, A., \& Mangal, N. S. (2013). Hyperprolactinemia. Journal of Human Reproductive Sciences, 6(3), 168-175. doi: 10.1007/978-81-322-1686-5_29

Nishimura, K., Matsumiya, K., Tsuboniwa, M., Koga, M., Miura, H., Tsujimura, A., Uchida, K., Kondoh, N., Kitamura, M., \& Okuyama, A. (1999). Bromocriptine for infertile males with mild hyperprolactinemia: hormonal and spermatogenic effects. Archives of Andrology, 43, 207-213. doi: 10.1080/014850199262517

Oldham, J. S., Zimmerman, J. B., \& Hotfield, B. D. (2019). Depression and anxiety disorders. In: Moore, G. E, Durstine, J. L., \& Painter, P. L. (eds). Exercise management for persons with chronic disease and disabilities USA, American college of sports medicine: Human Kinetics (293-300). doi: 10.1249/01. mss.0000320868.98282.42

Page, J., \& Thelwell, R. (2013). The value of social validation in single case methods in sport and exercise psychology. Journal of Applied Sport Psychology, 25(1), 61-71. doi: 10.1080/10413200.2012.663859

Palubska, S., Adamiak-Godlewska, A., Winkler, I., Romanek-Piva, K., Rechberger, T., \& Gogacz, M. (2017). Hyperprolactinemia - a problem in patients from the reproductive period to the menopause. Menopause Reviw, 16(1), 1-7. doi: 10.5114/pm.2017.67364

Panidis, D., Rousso, D., Skiadopoulos, S., Panidou, E., \& Mamopoulos, M. (1997). Evaluation of semen parameters in men with hyperprolactinemia induced by Metoclopramide. Archives of Andrology, 39, 237242. doi: $10.3109 / 01485019708987922$

Raveendranthan, D., Rao, N. P., Rao, M. G., Mangot, A. G., Varambally, S., Kesavan, M., Venkatasubramanian, G., \& Gangadhar, B. N. (2018). Add-on Aripiprazole for atypical antipsychotic-induced-clinically significant hyperprolactinemia. Indian Journal of Psychological Medicine, 40(1), 38-40. doi: 10.4103/ IJPSYM.IJPSYM_147_17

Sharma, N., Dutta, D., \& Sharma, L. K. (2017). Hyperpolactinemia in children with subclinical hypothyroidism. Journal of Clinical Research in Pediatric Endocrinology, 9(4), 350-354. DOI: 10.4274/jcrpe.4536

Sperling, S., \& Bhatt, H. (2016). Prolactinoma: a massive effect on bone mineral density in a young patient. Case Reports in Endocrinology, 2016, 1-3. doi: 10.1155/2016/6312621

Tirgar-Tabari, S., Sharbatdaran, M., Manafi-Afkham, S., \& Montazeri, M. (2016). Hyperprolactinemia and hirsutism in patients without polycyctic ovary syndrome. International Journal of Trichology, 8(3), 130134. doi: 10.4103/0974-7753.188998

Toulis, K. A., Robbins, T., Reddy, N., Balachandra, K., Gokhale, H., Cheng, K. K., Karavitaki, N., Wass, J., \& Nirantharakumar, K. (2018). Males with prolactinoma are at increased risk of incident cardiovascular disease. Clinical Endocrinology, 88, 71-76. DOI: 10.1111/cen.13498

Yamashita, M., \& Yamamoto, T. (2014). Tryptophan and kynurenic acid may produce an amplified effect in central fatigue induced by chronic sleep disorder. International Journal of Tryptophan Research, 7, 9-14. doi: 10.4137/IJTR.SI4084

Yancar-Demir, E., \& Sayin, A. (2014). Prolongation of hyperprolactinemia by Clozapine: a case report. Bulletin of Clinical Psychopharmacology, 24(4), 371-375. doi: 10.5455/bcp.20131127065702 